\title{
Research on Self-organization Evolution of Supply Chain Complex System Fulfilling Social Responsibility
}

\author{
Baoying Wang ${ }^{1,2}$ \\ ${ }^{1}$ School of management and economics,Beijing institute of technology,Beijing,100081,China \\ ${ }^{2}$ College of economics and management,North university of China,Taiyuan, 030051,China \\ E-mail: baoying_wang@nuc.edu.cn
}

Keywords:supply chain complex system;corporate social responsibility; self-organization evolution;systematic dynamics

\begin{abstract}
Based on the Self-organization characteristics of supply chain complex system,the systematic dynamics model is used to analyse the self-organization evolution when supply chain complex system fulfilling social responsibility.The research results find the self-organization evolution when supply chain complex system fulfilling social responsibility is related to the system's flows,the degree of dependence among the upstream and downstream enterprises and external environment factors. The research demonstrates that every sub-system of supply chain complex system can produce synertistic effect through coordination and fulfill social responsibility better.
\end{abstract}

\section{Introduction}

Accompanying the great achievements of economic construction in China since reform and opening-up,more economic,society and environment issues have exposed.Some enterprises do not undertake their social responsibility actively,including economic,legal,ethical and discretionary responsibilities.The government,society and consumers' sanctions and supervisions are not the driving force of fulfillment of social responsibility.We have to rethink the reasons why the enterprises challenge the bottom line of ethics and harm the interests of the masses under the background of Chinese improving legal environment,close government supervision and public opinion tremendous force.It is easy to recognize more enterprises act independently but not link with their supply chain when fulfilling social responsibility. They are often suffered by other enterprise's bad behaviors.

So it is necessary to analyse the self-organization evolution of supply chain complex system based on self-organization characteristics and using systematic dynamics. Through the analysis,we would like to find the related factors of evolution and further improve the social responsibility level.All of these provide certain theoretical guide and practical help for supply chain enterprises in fulfilling social responsibility.

\section{The Self-organization Characteristics of the Supply Chain Complex System}

The study define the supply chain complex system consists of three node enterprises,that is supplier,manufacturer and seller and name the supply chain complex system as the SCCS.It is a three stages supply chain,and the supplier,manufacturer and seller are the sub systems of SCCS.The basis of cooperation is their mutual benefit.They can get competitive advanges through improving the relationship of upstream and downstream and integrating and optimizing the information flow,material flow and capital flow among SCCS.

The self-organization theory is a systematic theory founded and developed since the late 1960's.Its research subject is mainly the formation and development of complex selforganization.That is, under certain conditions, how the system evolve from chaos to order,from the lower order to higuer order. The self-organization is a common phenomenon in nature and the society.The more stronger of self-organization function of system,the more stronger capability which produce and maintain new function.It consists of and focuses Ilya Prigogine's dissipative 
structure theory,Haken's synergistic theory, Eigen.Manfred's hypercycle theory and Rene Thom's catastrophe theory and chaos theory.The basic thought and theory core are represented by dissipative structure theory and synergistic theory[1][2].

The SCCS have clear characteristics of self-organization including opening,far-from equilibrium,non-linear and fluctuation.

\section{The Opening of the SCCS}

The SCCS exchanges materials,energies and informations with external environment. The system's boundary have certain opening characteristics. The external environment includes social environment and natural environment.The system acquires elements from the external environment,such as production materials,land,equipment,and return,gets related imformation,such as product information,price information,technology information,competitor's information,policy and law's information.The system provides the market with product,service and job opportunities and pays taxes.All of these embody the system's opening characteristics. Certainly,the system expects the enterprises fulfill social responsibility actively,and the enterprises also treat it as their operating goals. This is an interactive process[3].

The Far-from-equilibrium of the SCCS

The self-organization theory consider that the prerequisite of system's evolution is far from equilibrium and in a non-equilibrium. Non-equilibrium is the source of order.As a opening system, the interior of SCCS presents the characteristics of far from even and single through the exchanges of materials,energies and informations with external environment.It is a active state which have certain regularity and good circulation state. The system reaches non-equilibrium through two kinds input of entropy:system positive entropy change and system negative entropy change.The processes of operation and management are to make system total entropy change be less than zero.The introduction of negative entropy can make the system be far from equilibrium and develop towards non-equilibrium,meanwhile,adjust by itself and strengthen self-organization abilities[4].

The Non-linear of the SCCS

The relationship among the sub-systems is not a simple cause-effect or superposition relationship,but a kind of complex corelationship.In the system,the function of every factor and subsystem is not even and symmetric.The change of every factor and sub-system can cause the change of other factors or sub-systems and influence the whole system's development and change[5]. The research results of self-organization show that the interaction of inside system and outside system are both non-linear.

The Fluctuation of the SCCS

The fluctuation is the deviation from the system's stable and average state.It can destroy the system's stability and build the new stability.According to the research of synergetics, There are no random fluctuations in the system,there are no development.The existing differences among systematic factors or sub-systems lead to the deviation and form the fluctuation.The favorable fluctuation can cause the other factors or sub-systems' responses and relatedness effect,magnify it to huge fluctuation.Thus, the system form a more stable and order situation.

As analysed above,the SCCS have the characteristics of self-organization.So the system can achieve the self-organization evolution and could achieve a high level order situation in fulfilling social responsibility.

\section{The Self-organization Systematic Dynamics Model Establishment of the SCCS in Fulfilling Social Responsibility}

Basic Hypotheses

Assuming there are four time factor variables.

The interaction strength which the SCCS fulfills social responsibility is $S(t)$,indicates the stability of the SCCS's social responsibility.

The degree of dependence of every sub-systems in the SCCS is $D(t)$,includes product,technology,brand and channel dependence[6].

The systematic flow of materials,energies and informations is $F(t)$. 
The influence variables of external environment in the SCCS's fulfilling social responsibility is $E(t)$.

Generally, the above four variables can be regarded as the variables which influence the SCCS's social responsibility level,and $S(t), D(t), F(t)$ are the system's internal variables, $E(t)$ is the external variable.The fluctuations which the SCCS fulfills social responsibility are $\Delta S, \Delta D, \Delta F$ and $\Delta E$ respectively[7].

The Variables Analysis of The SCCS Self-organization Evolution Model When Fulfilling Social Responsibility

$S(t)$ depends on the two factors, the systematic flow of materials,energies and informations $F(t)$ and the degree of dependence of every sub-systems $D(t)$. The more the flow $F(t)$ and the higher the degree of dependence $D(t)$, the better fulfilling social responsibility. $F(t)$ and $D(t)$ have the positive influences on the stability of the SCCS's social responsibility level.That is,

$$
S(t)=f(F, D)
$$

In equation (1), $\frac{\partial f}{\partial F}>0, \frac{\partial f}{\partial D}>0$.

$D(t)$ and $S(t)$ are corrective. When the sub-systems have close cooperation,their dependence degree will increase.The increasing speed of the dependence degree will weaken following the increase of the stability of the SCCS.That is,

$$
D^{\prime}(t)=g(S, D)
$$

In equation (2), $\frac{\partial g}{\partial S}>0, \frac{\partial g}{\partial D}<0$.

$F(t)$ depends on $D(t)$ and $E(t)$.That is,

$$
F^{\prime}(t)=h(D, F ; E)
$$

In equation (3), $\frac{\partial h}{\partial D}<0, \frac{\partial h}{\partial F}<0, \frac{\partial h}{\partial E}>0$.

From equation (2) and (3), the change rate of $D(t)$ can be expressed as the function of $F(t)$ and $S(t)$.When $F(t)$ increases gradually, the $D(t)$ will also increase gradually. That is,

$$
D^{\prime}(t)=g(S, D)=h(F, D)
$$

$$
\text { In equation (4), } \frac{\partial h}{\partial F}=\frac{\partial g}{\partial S} \frac{\partial f}{\partial F}>0 \text { and } \frac{\partial h}{\partial D}=\frac{\partial g}{\partial D}+\frac{\partial g}{\partial S} \frac{\partial f}{\partial D} \text {. }
$$

Because $\frac{\partial g}{\partial I}>0, \frac{\partial g}{\partial D}<0$ and $\frac{\partial f}{\partial D}>0$, the negative or positive of $\frac{\partial h}{\partial D}$ is not fixed.Equation (4) shows $D(t)$ may be not certain follow the differences of situation of the degree of dependence.

So the SCCS's social responsibility level will be a relative stable situation when $F(t)$ is a fluctuation situation of average value,but the external environment fluctuate randomly and will influence the fluctuation range of every sub-system,lead to the catastrophe and evolution[8].

Focus on Equation (4).If $F(t)$ is higher,it can lead to the increase of $D(t)$; If $D(t)$ is higher,it will lead to a variety of effects. $D(t)$ keeps relative stable or the interior of the sub-systems have the selfremaining function.

Generally,when $\frac{\partial h}{\partial D}>0$, the higher the $D(t)$, the stronger its increase tendency;Contrarily,the weaker its decrease tendency.On the basis of equation (2),(3) and (4),two conclusions can be drown. 
Because $\frac{\partial g}{\partial I}>0$, the more higher the SCCS's social responsibility level,the higher the system stability,and the more stronger the degree of dependence of sub-system,and vice versa.

Bacause $\frac{\partial h}{\partial D}>0$,the flow size's increasing speed of materials,energies and informations will deccrease following increase of the degree of dependence of sub-system.

\section{The Mechanism of the SCCS's Self-organization Evolution When Fulfilling Social Responsibility}

The existence of the SCCS and its fulfilling social responsibility must be maintained by a sizable flows of materials,energies and informations.If the flows are very low or interruptive,the supply chain will broke down.Thus,each enterprise will fulfill social responsibility sepreately with bad effct.So it is necessary to analyse the stability of the SCCS's social responsibility level[9].

In the SCCS,the degree of dependence of every sub-system $D(t)$ is expressed as the system's degree of stability of social responsibility $S(t)$. The higher the $D(t)$, the more the $F(t)$, the higher the $S(t)$.This shows the size of flow and the probability of forming flow among the subsystems. The change of $S(t)$ and $D(t)$ can be expressed by the change of average flow in the system within a certain period.

In the SCCS,the factors which influence on the size of flow include internal factors and external factors.The internal factors include the sub-system's size change,the social responsibility's awareness and behavior improvement, the cost level's change and the market demand's change.Let $f_{i}$ express the degree of dependence under the action of internal factors. The external factors include the macroeconomic policy's change,the punishment and regulation to lack of social responsibility,the social groups and institutions' appeal,the media's extensive publicity and the consumer's more demanding.Let $f_{e}$ express the fluctuation of fulfilling social responsibility caused by the external factors[10][11].

Assume the flow size in the SCCS is non-linear and continuous distribution.Thus,the SCCS's flow size at a certain time can be expressed as follows.

$$
F(t)=\int_{t-1}^{t} F^{\prime}(t) d t+f_{e}(t)=\int_{t-1}^{t} h(t) d t+f_{e}(t)
$$

$F(t)$ is continuous with $[0, T]$, the density function is $h(t), F(t)$ is the flow size of time $t(t \leq T), \bar{F}_{t}$ is the average flow size within $[0, t](t \leq T)$. Thus,

(6)

$$
\overline{F_{t}}=\frac{\sum_{t} F_{t}}{t}=\frac{1}{t} \int_{0}^{t} h(t) d t+\overline{f_{e}}(t)
$$

$$
\Delta F_{t}=F_{t}-\overline{F_{t}}=\int_{t-1}^{t} h(t) d t-\frac{1}{t} \int_{0}^{t} h(t) d t+\Delta f_{e}
$$

Assume $f_{i}$ can express the $D(t)$ 's influence on the SCCS's self-organization evolution in fulfilling social responsibility,so within $[0, T]$,the SCCS's dependence factor $f_{i}^{t}$ at time $t$ can be regarded as the ratio $\Delta F$ and $\bar{F}$ at time $t(0 \leq t \leq T)$. That is, 
(8)

$$
f_{i}^{t}=\frac{\Delta F_{t}}{\bar{F}_{t}}=t \frac{\int_{t-1}^{t} h(t) d t}{\int_{0}^{t} h(t) d t}-1
$$

Set $\frac{\int_{t-1}^{t} h(t) d t}{\int_{0}^{t} h(t) d t}$ as $m$,the above (8) can be expressed as follows.

(9)

$$
f_{i}^{t}=m t-1
$$

$$
0 \leq t \leq T
$$

Equation (5) and (8) describe the interdependent relationship between the two system parameters.It is the basis which the SCCS evolves into self-organization when fulfilling social responsibility,it is also the power source of evolution[12][13].

\section{Conclusion}

The SCCS fulfilling social responsibility is a complex process.In this process, the formation and evolution of self-organization play an important role.The interaction strength which the SCCS fulfills social responsibility depends on the two factors,the systematic flow of materials,energies and informations and the degree of dependence of every sub-system.The more the flow and the higher the degree of dependence,the better coordination and fulfilling social responsibility. Meanwhile,the SCCS's evolution can not do without external environment.The changes of external environment will lead to the SCCS change.The government's control strategies,the social public opinion's supervision,the NGO's appeals and the consumer's demands can influence the improvement of the SCCS's fulfilling level social responsibility.In brief,from the angle of the SCCS, promoting the system forming self-organization through the influences of internal and external factors can make the SCCS the system evolve from chaos to order,from the lower order to higher order in fulfilling social responsibility.Based on the above,the further research will focus on the system's order parameters in fulfilling social responsibility and the coordination and profit sharing model in the future.

\section{Acknowledgment}

This research is supported by my academic adviser, professor Li Jian.Thanks for his responsibility and patience. The author would like to thank the other authors provided resources.

\section{References}

[1]Prigogine I,Alan P M, "The Chanllege of Complexity,Self-organization and Dissipative Structure”,University of Texas Press, 1982,pp.85-92.

[2]Haken H, “Information and Self organization”,Sichuan Education press,1988,pp.101-106.

[3]Xu Lida, Fan Ying, and Di Zengru, "Concepts,methods and applications of self-organization theory”,Journal of university of Shanghai for science and technology, vol.33,No. 2,2011,pp 130-136. [4]Gao Chunfeng, “The study for rural community development in self-organization theory”, China agricultural university press,2009,pp 128-130.

[5]Faisal, Mohd. Nishat, "Analysing the barriers to corporate social responsibility in supply chains: an interpretive structural modelling approach”, International Journal of Logistics-research and Applications,vol.13,No,3,2010,pp 179-195.

[6]Wei Dong, Yue Jie, “A study on corporate environmental responsibility based on selforganization theory”,Science and management, vol.4,2010,pp 26-29.

[7]Smith, N. Craig,"Marketing's Consequences: Stakeholder Marketing and Supply Chain Corporate Social Responsibility Issues”, Business Ethics Quarterly,vol.20,No.4,2010,pp 617-641.

[8]Salam, Mohammad Asif, “Corporate social responsibility in purchasing and supply Chain”, 
Journal of Business Ethics,vol 85,2009,pp 355-370.

[9]Risso M, “A horizontal approach to implementing corporate social responsibility in international supply chains”, International Journal of Technology Management,vol.58,2012,pp 64-82.

[10]Chi. T, "Building a sustainable supply chain: an analysis of corporate social responsibility (CSR) practices in the Chinese textile and apparel industry", Journal of the Textile Institute,vol.102,No.10,2011,pp 837-848.

[11]Tate, Wendy L.Ellram, and Lisa M.Kirchoff, Jon F, “Corporate social responsibility reports: a thematic analysis related to supply chain management", Journal of Supply Chain Management,vol.46,No.1,2011,pp 19-44.

[12]Huang Xiaowei,He Mingsheng,"Self-organization evolution of supply chain resources synergy”,Journal of Nanjing of Nanjing University of Science and Technology”,vol. 34,No.1,2010,pp 35-38.

[13]Cruz, Jose M, “The impact of corporate social responsibility in supply chain management: Multicriteria decision-making approach”, Decision Support Systems,vol.48,No.1,2009,pp 224-236. 